\title{
DENOMINATOR SEQUENCES OF CONTINUED FRACTIONS II
}

\author{
R. T. WORLEY \\ (Received 4 October 1971; revised 3 December 1971) \\ Communicated by E. S. Barnes
}

In part I, I considered the problem of discovering when, given an irrational $\alpha$ which has a simple continued fraction representation with convergents $p_{n} / q_{n}$, there exists $\alpha^{\prime}$ for which the denominator sequence for convergents is a subsequence of $\left(q_{n}\right)$. It was shown that such an $\alpha^{\prime}$ exists if the continued fraction representation was "nearly periodic" with odd period. The following is a generalization of the results of part I to semi-regular continued fractions, where the problem seems to fit more naturally.

Let $a_{0}, \varepsilon_{1}, a_{1}, \varepsilon_{2}, \cdots, \varepsilon_{n}, a_{n}$ be integers satisfying (i) $\varepsilon_{i}= \pm 1$ for $1 \leqq i \leqq n$, and (ii) $a_{i} \geqq 1$ for $1 \leqq i \leqq n$. If the repeated fraction

$$
a_{0}+\varepsilon_{1} /\left(a_{1}+\varepsilon_{2} /\left(\cdots /\left(a_{n-1}+\varepsilon_{n} / a_{n}\right) \cdots\right)\right)
$$

can be evaluated as a real number without dividing by zero we say that the symbol $\left\langle a_{0}, \varepsilon_{1}, a_{1}, \cdots, a_{n}\right\rangle$ is defined and is equal to that real number. It can be shown that division by zero never arises if $a_{i}+\varepsilon_{i+1} \geqq 1$ for $0 \leqq i \leqq n-1$.

By the infinite semi-regular continued fraction $\left\langle a_{0}, \varepsilon_{1}, a_{1}, \cdots\right\rangle$ is meant $\lim _{n \rightarrow \infty}\left\langle a_{0}, \varepsilon_{1}, a_{1}, \cdots, a_{n}\right\rangle$, where in writing $\left\langle a_{0}, \varepsilon_{1}, a_{1}, \cdots\right\rangle$ it is assumed that $\left\langle a_{0}, \varepsilon_{1}, a_{1}, \cdots, a_{n}\right\rangle$ is defined for all $n$ and $a_{i}+\varepsilon_{i+1} \geqq 1$ for all $i$. It is wellknown that the limit exists, and is irrational iff $\left.a_{i}+\varepsilon_{i+1}\right\rangle 1$ infinitely often.

It is also well-known that for all $n \geqq 1,\left\langle a_{0}, \varepsilon_{1}, \cdots, a_{n}\right\rangle=p_{n} / q_{n}$ where $p_{n}$ and $q_{n}$ are defined inductively by the properties

$$
\begin{array}{ll}
p_{0}=a_{0}, q_{0}=1, p_{1}=a_{1} a_{0}+\varepsilon_{1}, q_{1}=a_{1}, \\
p_{n}=a_{n} p_{n-1}+\varepsilon_{n} p_{n-2} & (n \geqq 2), \\
q_{n}=a_{n} q_{n-1}+\varepsilon_{n} q_{n-2} & (n \geqq 2) .
\end{array}
$$

This notation will be preserved for the rest of this paper. In addition, for convenience, we shall take $a_{0} \geqq 2$ in order to avoid difficulties with symbols $\left\langle\cdots, a_{0}\right\rangle$, though plainly the denominators $q_{i}(i \geqq 0)$ are independent of $a_{0}$. We define $\left\langle a_{0}, \varepsilon_{1}, a_{1}, \cdots\right\rangle$ to be nearly periodic with period $(p, r)$ if $p, r$ are non-negative integers with $p>1$ such that for each $n \geqq 1$ at least one of the following two 
equations holds, where the expressions are considered as (2p-3)-tuples

(i) $\left(a_{n p+r}, \varepsilon_{n p+r}, a_{n p+r-1}, \cdots, \varepsilon_{(n-1) p+r+3}, a_{(n-1) p+r+2}\right)$

$$
=\left(a_{n p+r+2}, \varepsilon_{n p+r+3}, a_{n p+r+3}, \cdots, a_{(n+1) p+r}\right)
$$

(ii) $\left(a_{(n-1) p+r+2}, \varepsilon_{(n-1) p+r+3}, \cdots, a_{n p+r-1}, \varepsilon_{n p+r}, a_{n p+r}\right)$

$$
=\left(a_{n p+r-2}, \varepsilon_{n p+r+3}, a_{n p+r+3}, \cdots, a_{(n+1) p+r}\right) \text {. }
$$

Thus "nearly periodic" is independent of the initial terms as there is no restriction on the size of $r$. The condition allows the numbers $\varepsilon_{n p+r+1}, a_{n p+r+1}, \varepsilon_{n p+r+2}$ to be arbitrary within the restrictions on a semi-regular continued fraction, while the blocks between these groups of three elements must recur in the same or reverse order.

Because we shall be reversing the order of elements of continued fractions we need the following lemma.

LeMma 1. Let $\left\langle a_{0}, \varepsilon_{1}, a_{1}, \cdots\right\rangle$ be a semi-regular continued fraction and let, for non-negative integers $r, n$ with $n \geqq r$,

$$
\left(b_{0}, \rho_{1}, b_{1}, \rho_{2}, \cdots, b_{r}\right)=\left(a_{n}, \varepsilon_{n}, a_{n-1}, \varepsilon_{n-1}, \cdots, \varepsilon_{n-\cdot+1}, a_{n-r}\right)
$$

as $(2 r+1)$-tuples. Then $\left\langle b_{0}, \rho_{1}, b_{1}, \cdots, b_{r}\right\rangle$ is defined and is positive. Furthermore it is not greater than 1 if and only if $r=0$ and $b_{0}=1$ or $r>0, b_{0}=1$ and $\rho_{1}=-1$.

PRoof. The result is trivial for $r=0$. If $r=1$ then $\left\langle b_{0}, \rho_{1}, b_{1}\right\rangle=a_{n}+\varepsilon_{n} \mid a_{n-1}$ is defined. If $\varepsilon_{n}=1$ then plainly $\left\langle b_{0}, \rho_{1}, b_{1}\right\rangle>a_{n} \geqq 1$. If $\varepsilon_{n}=-1$ and $a_{n} \geqq 2$ then since $a_{n-1} \geqq 1-\varepsilon_{n}=2$ we have $\left.\left\langle b_{0}, \rho_{1}, b_{1}\right\rangle \geqq 3 / 2\right\rangle 1$. If $\varepsilon_{n}=-1$ and $a_{n}=1$ then $a_{n-1} \geqq 2$ again and

$$
0<\frac{1}{2} \leqq 1-a_{n}^{-1}<1 .
$$

This proves the lemma for $r=1$. The proof is now completed by induction Assume truth for $r=k$. Then

$$
\left\langle b_{0}, \rho_{1}, b_{1}, \cdots, b_{k+1}\right\rangle=b_{0}+\rho_{1} /\left\langle b_{1}, \rho_{2}, \cdots, b_{k+1}\right\rangle
$$

and is defined since $\left\langle b_{1}, \cdots, b_{k+1}\right\rangle>0$ by assumption. In addition, $\left\langle b_{1}, \cdots, b_{k+1}\right\rangle$ $\leqq 1$ only if $b_{1}=1$, i.e. $a_{n-1}=1$. Then $\varepsilon_{n} \geqq 1-a_{n-1}=0$ and so $\varepsilon_{n}=1$, i.e. $\rho_{1}=1$. In this case $\left\langle b_{0}, \rho_{1}, b_{1}, \cdots, b_{k+1}\right\rangle \geqq 2$. Otherwise $\left.\left\langle b_{1}, \cdots, b_{k+1}\right)\right\rangle 1$ and so (i) if $\rho_{1}=1$ we have $\left.\left\langle b_{0}, \cdots, b_{k+1}\right\rangle\right\rangle 1$,

(ii) if $\rho_{1}=-1$ we have $b_{0}-1<\left\langle b_{0}, \cdots, b_{k+1}\right\rangle \leqq b_{0}$

and so $\left\langle b_{0}, \cdots, b_{k+1}\right\rangle \leqq 1$ if and only if $b_{0}=1$ and $\rho_{1}=-1$. This completes the induction step.

Another lemma that is easily proved by induction is

LEMMA 2. Let $\left\langle a_{0}, \varepsilon_{1}, a_{1}, \cdots\right\rangle$ be an infinite semiregular continued fraction 
and let $r, s$ be non-negative integers with $s>r$. Then

$$
\left\langle a_{r}, \varepsilon_{r+1}, a_{r+1}, \cdots, a_{s}\right\rangle \geqq 1
$$

with equality if and only if $a_{r}, a_{r+1}, \cdots, a_{s-1}$ are all $2, \varepsilon_{r+1}, \cdots, \varepsilon_{s}$ are all -1 and $a_{s}=1$.

The following lemmas are the natural generalization of the results of part I and are proved in the same manner.

LEMma 3. Let $k, l, m$ be positive integers with $m>l$. Define $P_{m}, Q_{m}, R_{m}, S_{m} b y$

$$
\begin{aligned}
& P_{m} / Q_{m}=\left\langle a_{m+1}, \varepsilon_{m+2}, a_{m+2}, \cdots, a_{m+k}\right\rangle \\
& R_{m} / S_{m}=\left\langle 0,1, a_{m}, \varepsilon_{m}, a_{m-1}, \cdots, a_{m-l+2}\right\rangle
\end{aligned}
$$

where the second is interpreted as $0 / 1$ if $l=1$ and is defined by lemma 1 . Then

$$
\begin{gathered}
q_{m+k}=P_{m} q_{m}+\varepsilon_{m+1} Q_{m} q_{m-1}, \\
\varepsilon_{m} \varepsilon_{m-1} \cdots \varepsilon_{m-l+2} q_{m-1}=(-1)^{l-1}\left(S_{m} q_{m-1}-R_{m} q_{m}\right),
\end{gathered}
$$

and similarly with the $q_{i}$ replaced by $p_{i}$.

COROLlARY. If $\left\langle a_{0}, \varepsilon_{1}, a_{1}, \cdots\right\rangle$ is nearly periodic with period $(p, r)$ then for all $n \geqq 1$,

$$
\begin{aligned}
& q_{(n+1) p+r}=c_{n+1} q_{n p+r}+\eta_{n+1} q_{(n-1) p+r} \\
& p_{(n+1) p+r}=c_{n+1} p_{n p+r}+\eta_{n+1} p_{(n-1) p+r}
\end{aligned}
$$

where

$$
\begin{aligned}
& c_{n+1}=P_{n p+r}+\varepsilon_{n p+r+1} R_{n p+r} \\
& \eta_{n+1}=(-1)^{p-1} \varepsilon_{n p+r+1} \varepsilon_{n p+r} \cdots \varepsilon_{(n-1) p+r+2} .
\end{aligned}
$$

It should be noted that the first part of the conclusion of Lemma 1 is basically Perron [1] p. 15 eq. (24) while the result, for $\left\langle a_{0}, \varepsilon_{1}, a_{1}, \cdots\right\rangle$ nearly periodic with period $(d, p, r)$, that $Q_{n p+r}=S_{n p+r}$, is basically Perron p. 12 equation (18). The following lemma can be proved easily by induction.

LEMMA 4. Let $d_{2}, \cdots, d_{s}$ be positive integers, let $\rho_{2}, \cdots, \rho_{s}$ satisfy $\rho_{i}= \pm 1$ $(2 \leqq i \leqq s)$, let $X_{0}, X_{1}, Y_{0}, Y_{1}$ be non-negative integers and let $X_{2}, \cdots, X_{s}$ $Y_{2}, \cdots, Y_{s}$ be defined inductively by

Then

$$
\begin{aligned}
& X_{m}=d_{m} X_{m-1}+\rho_{m} X_{m-2} \quad(2 \leqq m \leqq s) . \\
& Y_{m}=d_{m} Y_{m-1}+\rho_{m} Y_{m-2}
\end{aligned}
$$

$$
X_{s} / Y_{s}=\left(X_{1} \beta_{s}+\rho_{2} X_{0}\right) /\left(Y_{1} \beta_{s}+\rho_{2} Y_{0}\right)
$$

where $\beta_{s}=\left\langle d_{2}, \rho_{3}, d_{3}, \cdots, \rho_{s}, d_{s}\right\rangle$. 
The following theorem and its corollary are the main results.

THEOREM. Let $\alpha=\left\langle a_{0}, \varepsilon_{1}, a_{1}, \cdots\right\rangle$ be such that for positive integers $c_{2}, c_{3}, c_{4}, \cdots$

(i) $q_{i_{n+1}}=c_{n+1} q_{i_{n}}+\eta_{n+1} q_{i_{n-1}} \quad(n \geqq 1)$, and

(ii) $p_{i_{n+1}}=c_{n+1} p_{i_{n}}+\eta_{n+1} p_{i_{n-1}} \quad(n \geqq 1)$

for a subsequence $\left(q_{i_{n}}\right)$ of $\left(q_{i}\right)$, where $\eta_{i}= \pm 1$ and $c_{i}+\eta_{i+1} \geqq 1$ for $i \geqq 2$ and $\eta_{2}=1$ if $q_{i_{1}} \leqq q_{i_{0}}$.

If $q_{i_{\mathrm{n}}}$ and $q_{i_{1}}$ are relatively prime then there exists $\alpha^{\prime}$ of the form $a \alpha+b$ with $a$ and $b$ rational such that $\alpha^{\prime}$ has a semi-regular continued fraction expansion for which the convergents are $A_{n} / B_{n}$ with $B_{n+u}=q_{i_{n}}$ for $n \geqq 0$, where $u \geqq 0$ is integral and $u=0$ if $q_{i_{0}}=1$.

PROOF. If $q_{i_{0}}=1$ then plainly the denominators of convergents to $\alpha^{\prime}=\left\langle 0,1, q_{i_{1}}, \eta_{2}, c_{2}, \eta_{3}, c_{3} \cdots\right\rangle$ are $q_{i_{0}}, q_{i_{1}} \cdots$. However if $q_{i_{0}}>1$ define $q_{i-1}, q_{i-2}, \cdots$ and $c_{1}, c_{0}, c_{-1}, \cdots$ inductively by

$$
q_{i_{j+2}}=c_{j+2} q_{i_{j+1}}+\eta_{j+2} q_{i_{j}}\left(j \leqq-1 ; 0 \leqq q_{i_{j}} \leqq q_{i_{j+1}}-1\right)
$$

where if $q_{i_{1}}>2 q_{i_{0}}$ then $\eta_{j+2}$ is always chosen as +1 and if $q_{i_{1}}<2 q_{i_{0}}$ then $\eta_{j+2}$ is always chosen as -1 . The process is considered to stop when $q_{i_{t}}=0$ is first reached. It is easy to verify that the convergents to

$$
\alpha^{\prime}=\left\langle 0,1, c_{t+2}, \eta_{t+2}, c_{t+3}, \cdots\right\rangle
$$

have denominators $q_{i_{t+1}}, q_{i_{t+2}}, \cdots$.

To find the relation between $\alpha$ and $\alpha^{\prime}$ we observe that by lemma 4

$$
\alpha=\lim _{n \rightarrow \infty} p_{i_{n}} / q_{i_{n}}=\left(p_{i_{1}} \beta+\eta_{2} p_{i_{0}}\right) /\left(q_{i_{1}} \beta+\eta_{2} q_{i_{0}}\right)
$$

where $\beta=\left\langle c_{2}, \eta_{3}, c_{3}, \cdots\right\rangle$. But we have, by Perron p. 7 eq. (11),

$$
x^{\prime}=\left(y \beta+\eta_{2} x\right) /\left(q_{i_{1}} \beta+q_{i_{0}} \eta_{2}\right)
$$

where $y / q_{i_{1}}=\left\langle 0,1, c_{t+2}, \varepsilon_{t+3}, \cdots, c_{1}\right\rangle$ and $x / q_{i_{0}}=\left\langle 0,1, c_{t+2}, \cdots, c_{0}\right\rangle$, these expressions being interpreted as $1 / q_{i_{1}}$ and $0 / 1$ if $q_{i_{0}}=1$. Comparing the above formulae gives

$$
\alpha^{\prime}=\left(p_{i_{1}} q_{i_{0}}-p_{i_{0}} q_{i_{1}}\right)^{-1}\left(\alpha\left(y q_{i_{0}}-x q_{i_{1}}\right)+x p_{i_{1}}-y p_{i_{0}}\right)
$$

which is clearly of the desired form. It remains to set $u=-(t+1)$.

It will be noticed that if $\left(q_{i_{1}}, q_{i_{0}}\right)=d \neq 1$ then carrying out the above procedure on $d^{-1} q_{i_{0}}$ and $d^{-1} q_{i_{1}}$ gives $\alpha^{\prime}$ as above, the denominators of the convergents being $d^{-1} q_{i_{t+1}}, d^{-1} q_{i_{t+2}}, \cdots$, and we have

$$
\begin{aligned}
\alpha^{\prime} & =\left(y \beta+\eta_{2} x\right) /\left(d^{-1} q_{i_{1}} \beta+d^{-1} q_{i_{0}} \eta_{2}\right) \\
& =a \alpha+b
\end{aligned}
$$

for rational $a$ and $b$, as before. 
COROLlary. If $\alpha$ is irrational with continued fraction expansion $\alpha=\left\langle a_{0}, \varepsilon_{1}, a_{1}, \cdots\right\rangle$ nearly periodic with period $(p, r)$, then

(a) If $r=0$ or $r=a_{1}=1$ then $q_{r}=1$ and the theorem with $i_{n}=n p+r$ yields the existence of $\alpha^{\prime}$ the denominators of convergents to which form $a$ subsequence $\left(B_{n}\right)=\left(q_{i_{n}+k p}\right)$, for a suitable integer $k$, of the denominator sequence of convergents to $\alpha$.

(b) If $\left(q_{r}, q_{p+r}\right)=1$ then the theorem with $i_{n}=n p+r+k p$ for a suitable integer $k$ yields $\alpha^{\prime}$ the denominators of convergents to an expansion of which, apart from an initial few, form a subsequence $\left(B_{n+u}\right)=\left(q_{i_{n}}\right)$ of the denominator sequence of convergents to $\alpha$.

(c) If $\left(q_{r}, q_{p+r}\right)=d>1$ then by the remarks above a similar conclusion holds except that the denominators of convergents to $\alpha^{\prime}$ need to be multiplied by $d$.

Proof. It is only necessary to show the conditions of the theorem are satisfied. The corollary to Lemma 3 is used. Plainly $\eta_{i}= \pm 1$. We must show that $c_{i}+\eta_{i+1} \geqq 1$ for $i \geqq 2$. Since $\eta_{n+2} \geqq-1$ and $c_{n+1} \geqq P_{n p+r}-R_{n p+r}$ for $n \geqq 1$ this condition is satisfied if $P_{n p+r} / Q_{n p+r}>1$ and $R_{n p+r} / S_{n p+r}<1$ for then $Q_{n p+r}=S_{n p+r}$ implies that $P_{n p+r} \geqq R_{n p+r}+2$. Now suppose that $P_{n p+r} / Q_{n p+r} \leqq 1$ By lemma 2 this implies that

$$
\left\langle a_{n p+r+1}, \varepsilon_{n p+r+2}, a_{n p+r+3}, \cdots, a_{(n+1) p+r}\right\rangle=\langle 2,-1,2, \cdots, 2,-1,1\rangle .
$$

But then

$$
\begin{aligned}
\eta_{n+2} & =(-1)^{p-1} \varepsilon_{n p+r+2} \varepsilon_{n p+r+3} \cdots \varepsilon_{(n+1) p+r} \varepsilon_{(n+1) p+r+1} \\
& =(-1)^{p-1}(-1)^{p-1} \varepsilon_{(n+1) p+r+1} \\
& =\varepsilon_{(n+1) p+r+1} \\
& \geqq 1-a_{(n+1) p+r}=0 .
\end{aligned}
$$

Thus $\eta_{n+2}=1$ and so $c_{n+1}+\eta_{n+2} \geqq 1$. Similarly if we suppose $R_{n p+r} / S_{n p+r} \geqq 1$ then using lemma 1 we have

$$
\left\langle a_{n p+r}, \varepsilon_{n p+r}, \cdots, a_{(n-1) p+r+2}\right\rangle=\langle 1,-1, \cdots\rangle \text { or }\langle 1\rangle \text {. }
$$

But $a_{n p+r}=1$ implies $\varepsilon_{n p+r+1} \geqq 1-1=0$, so $\varepsilon_{n p+r+1}=1$ and hence $c_{n+1}$ $=P_{n p+r}+\varepsilon_{n p+r+1} R_{n p+r} \geqq 1+1=2$, since neither $P_{n p+r} / Q_{n p+r}$ nor $R_{n p+r} / S_{n p+r}$ can be zero. It remains to satisfy the condition $\eta_{2}=1$ if $q_{i_{1}} \leqq q_{i_{0}}$. We do this by choosing $i_{n}=(n+k) p+r$ such that $q_{i_{1}}>q_{i_{0}}$. This is possible since $\alpha$ is assumed irrational and $\lim _{k \rightarrow \infty}\left|\alpha-p_{k p+r}\right| q_{k p+r} \mid=0$.

It will be noted that if a nearly periodic simple continued fraction expansion of $\alpha$ is chosen then the continued fraction expansion of $\alpha^{\prime}$ that is obtained is simple if the period is odd and the "integer above" expansion if the period is even. For this expansion we have, eventually, $c_{n+1}=P_{n p+r}+R_{n p+r}$ as all $\varepsilon$ are 1 . 
By lemmas 1 and 2 we have $P_{n p+r} / Q_{n p+r}>1$ and $R_{n p+r} / S_{n p+r} \neq 0$. Thus $P_{n p+r} \geqq 2$ and $R_{n p+r} \geqq 1$ and so $c_{n+1} \geqq 3$. The convergents $h / k$ to $\alpha^{\prime}$ corresponding to these $c$ therefore satisfy $\left|\alpha^{\prime}-h / k\right|<1 / 2 k^{2}$ and so by a well-known result are convergents of the simple continued fraction for $\alpha^{\prime}$. Thus $\alpha$ and $\alpha^{\prime}$ have simple continued fraction expansions with a common subsequence of denominators.

\section{References}

[1] O. Perron, Die Lehre von den Kettenbrüchen (Chelsea).

[2] R. T. Worley, 'Denominator Sequences of Continued Fractions I,' Aust. Math. Soc. 15 (1973), 112-116.

Monash University

Clayton, Victoria, 3168

Australia 